\title{
Experimental investigation of a mid-infrared Er:ZBLAN fiber laser
}

\author{
Łukasz Pajewski, ${ }^{1}$ Łukasz Sójka, ${ }^{* 1}$ Samir Lamrini, ${ }^{2}$ Trevor M. Benson, ${ }^{3}$ Angela B. Seddon ${ }^{3}$ and Sławomir Sujecki ${ }^{1,3}$ \\ ${ }^{I}$ Faculty of Electronics, Wroclaw University of Science and Technology, Wyb. Wyspianskiego 27, 50-370 Wroclaw \\ ${ }^{2}$ LISA laser products OHG, Max-Planck-Str. 1, Katlenburg-Lindau 37191, Germany \\ ${ }^{3}$ Faculty of Engineering, Unversity of Nottingham, University Park, Nottingham NG7 2RD, UK
}

Received April 08, 2020; accepted September 22, 2020; published September 30, 2020

\begin{abstract}
In this contribution the diode pumped high-power Er:ZBLAN laser operating at around $2.8 \mu \mathrm{m}$ is reported. The laser produces $2 \mathrm{~W}$ output power with the slope efficiency of $24 \%$ measured with respect to the incident pump power.
\end{abstract}

Fiber lasers have provided the key technology enabling many applications [1-3]. Fiber lasers can be characterized by good quality of the output beam, compact structure, high efficiency, excellent heat dissipation properties and very good reliability [1-3]. Most of the commercially available fiber lasers operate up to approximately $2 \mu \mathrm{m}$ of output wavelength. For example, the $\mathrm{Tm}^{3+}$ doped silica fiber laser can deliver more than $100 \mathrm{~W}$ of output power at $1.94 \mu \mathrm{m}$ [4]. However, there is large demand for fiber lasers operating in the spectral region beyond $2 \mu \mathrm{m}$, especially between 3 and $5 \mu \mathrm{m}$. This is because many important molecules have vibrational resonant frequencies within this spectral region to enable molecular sensing. To access wavelengths stretching between 3 and $5 \mu \mathrm{m}$, low phonon host materials are needed. Among the most promising and feasible low-phonon materials for realization of fiber laser operating beyond $3 \mu \mathrm{m}$ is the fluorozirconate glass [1]. ZBLAN (composed of fluorides: $\mathrm{ZrF}_{4}, \mathrm{BaF}_{2}, \mathrm{LaF}_{3}, \mathrm{AlF}_{3}, \mathrm{NaF}$ ) glass fibers have already proven their usefulness in the development of MIR (midinfrared) fiber lasers operating at around $3 \mu \mathrm{m}$ [1]. To date, mid-infrared laser action near $3 \mu \mathrm{m}$ has been observed in fluorozirconate glasses doped with $\mathrm{Er}^{3+}, \mathrm{Ho}^{3+}$ and $\mathrm{Dy}^{3+}$ [5-9]. It should be mentioned that those lasers can generate Watt level output powers. When compared with $\mathrm{Er}^{3+}$, pumping $\mathrm{Ho}^{3+}$ or $\mathrm{Dy}^{3+}$ doped glasses is more difficult. This is because for pumping $\mathrm{Ho}^{3+}$ doped glass laser diodes operating at $1.15 \mu \mathrm{m}$ are required, which are rather uncommon. Similarly, for pumping $\mathrm{Dy}^{3+}$ fiber lasers, laser diodes operating at $1.1 \mu \mathrm{m}, 1.3 \mu \mathrm{m}, 1.7 \mu \mathrm{m}$ or $2.8 \mu \mathrm{m}$ [6-9] are needed, which again are rather sparse, commercially. For comparison, the $\mathrm{Er}^{3+}$ :ZBLAN fiber laser which operates near $2.8 \mu \mathrm{m}$, utilizing the ${ }^{4} \mathrm{I}_{11 / 2} \rightarrow$ ${ }^{4} \mathbf{I}_{132} \quad$ transition, can be pumped at $0.975 \mu \mathrm{m}$ by easily commercially available,

*E-mail: lukasz.sojka@pwr.edu.pl high brightness laser diodes with hundreds of Watts of output power.

The highest to date achieved output power for $\mathrm{CW}$ (continuous wave) operation of erbium ion doped fluoride fiber lasers is $41.6 \mathrm{~W}$, under $0.975 \mu \mathrm{m}$ multimode diode pumping with a slope efficiency of $23 \%$ [5]. Achieving such output powers using fluoride fibers is, however, not easy due to fiber end facet degradation through $\mathrm{OH}$ diffusion under heating. Fast fiber end facet degradation is one problem occurring at such output power levels. It can be prevented by splicing $\mathrm{AlF}_{3}$ endcaps [5]. An alternative solution to the fiber end facet degradation is sputtering of a nanoscopic diffusion barrier film of silicon nitride $\left(\mathrm{Si}_{3} \mathrm{~N}_{4}\right)$ [10]. Furthermore, it was shown that the fiber end facet degradation issue can be improved by developing a ZBLAN-fiber-based side-pump combiner. Using this approach, the $\mathrm{Er}^{3+}: \mathrm{ZBLAN}$ fiber laser with an output power of $33 \mathrm{~W}$ was demonstrated in 2018 [11].

More recently, fiber lasers with emission wavelength above $3.5 \mu \mathrm{m}$ have been reported [12]. For example, a CW $3.92 \mu \mathrm{m}$ laser action at room temperature has been observed in $\mathrm{Ho}^{3+}$ doped fluoroindate glass fibers [13]. Also swept-wavelength $(2.8-3.4 \mu \mathrm{m}) \mathrm{Dy}^{3+}$ fluoride fiber lasers have proved useful for real time sensing of ammonia gas [14]. Moreover, it should be noted that, recently, a lot of attention was devoted to the development of co-doped $\mathrm{Er}^{3+} / \mathrm{Dy}^{3+}, \mathrm{Er}^{3+} / \mathrm{Ho}^{3+}, \mathrm{Er}^{3+} / \mathrm{Tm}^{3+}$ and $\mathrm{Dy}^{3+} / \mathrm{Tm}^{3+}$ fluorozirconate and fluoroindate glasses. These co-doped glasses may prove instrumental in developing novel, more efficient, high-power, diode-pumped fiber lasers operating at $3 \mu \mathrm{m}$ [15-18].

Finally, for the sake of completeness, it is noted that ZBLAN glass is also a very promising host material for the construction of visible light fiber lasers [19,20] and MIR supercontinuum sources [21].

In this letter, the lasing properties of the $\mathrm{Er}^{3+}$ : ZBLAN fiber laser are studied. The results show that more than $2.08 \mathrm{~W}$ of output power at around $2.8 \mu \mathrm{m}$ can be obtained with a slope efficiency of $24 \%$ when pumping a $\mathrm{Er}^{3+}$ : ZBLAN fiber laser at $0.975 \mu \mathrm{m}$.

Figure 1 presents a simplified energy level diagram of 
$\mathrm{Er}^{3+}$ in ZBLAN glass. MIR emission at around $2.8 \mu \mathrm{m}$ is expected from the ${ }^{4} \mathrm{I}_{11 / 2} \rightarrow{ }^{4} \mathrm{I}_{13 / 2}$ transition under excitation at $0.975 \mu \mathrm{m}$. The lifetime of the upper laser level ${ }^{4} \mathrm{I}_{11 / 2}$ was measured to be $6.9 \mathrm{~ms}$ [9]. It is noted that the lower laser level ${ }^{4} \mathrm{I}_{13 / 2}$ has a longer lifetime of around $9 \mathrm{~ms}$, which leads to self-saturation of laser action at $2.8 \mu \mathrm{m}$. Fortunately, in highly doped $\mathrm{Er}^{3+}: \mathrm{ZBLAN}$ this problem is solved by an energy-transfer up-conversion (ETU1) mechanism that efficiently depopulates the lower laser level ${ }^{4} \mathrm{I}_{13 / 2}$ [9]. For a large number of ions participating in ETU1 process it was predicted that a slope efficiency twice the Stokes limit can be achieved [9].

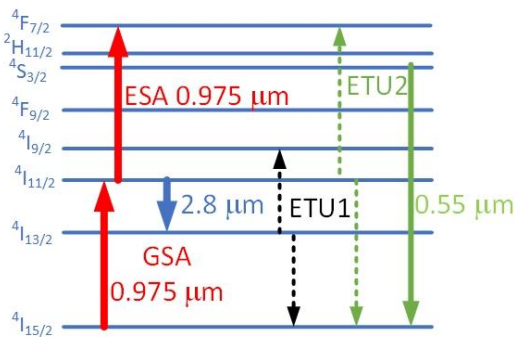

Fig. 1. Energy level diagram of trivalent erbium ions in ZBLAN glass (adopted from [9])

The experimental setup is shown in Fig. 2. The doubleclad $\mathrm{Er}^{3+}: \mathrm{ZBLAN}$ fiber used in the experiment was 3 meter long. The fiber had a 7 mol.\% core doping concentration of $\mathrm{ErF}_{3}$, a core diameter of $15 \mu \mathrm{m}$ and a cladding diameter of $260 \mu \mathrm{m}\left(\mathrm{NA}_{\text {core }}=0.12\right.$ (between fiber core and first clad), $\mathrm{NA}_{\text {clad }}=0.46$, (between first clad and second polymer clad) Le Verre Fluoré (NA is numerical aperture) with a pump absorption of approximately $3 \mathrm{~dB} / \mathrm{m}$ at $0.975 \mu \mathrm{m}$ [22]. Therefore, 3 meters of gain fiber was selected to ensure around $90 \%$ of pump absorption. The cladding glass of the fiber used had a double D-shape geometry which helped to improve pump absorption. The fiber ensured single transverse mode operation for wavelengths above $2.5 \mu \mathrm{m}$. The fiber was single mode for wavelengths above $2.5 \mu \mathrm{m}$. The fiber ends $\mathrm{A}$ and $\mathrm{B}$ were placed in stainless steel $\mathrm{V}$-grooves whilst the reaming part of the $\mathrm{Er}^{3+}$ :ZBLAN fiber was mounted to aluminum plate using a thermal conductive silicone gel pad in order to improve heat dissipation. Around $2 \mathrm{~mm}$ of input fiber end was protruding from a Vgroove and was flushed by pressurized dry-air in order to reduce moisture diffusion into the fiber tip. This approach also provides convection cooling of the fiber tip.

The $\mathrm{Er}^{3+}:$ ZBLAN fiber was pumped by $0.975 \mu \mathrm{m}$ multimode diode laser coupled to $105 \mu$ m multimode fiber (BWT K976DA3RN-30.00W). The collimated light from the $0.975 \mu \mathrm{m}$ pump was reflected by a dichroic mirror (highly reflective for wavelengths around $0.975 \mu \mathrm{m}$ and highly transmissive for wavelengths between 2.8-3.2 $\mu \mathrm{m}$ ). Then the pump light was focused into the fiber clad using an uncoated plano-convex $\mathrm{CaF}_{2}$ lens, with a focal length of $25 \mathrm{~mm}$. The fiber end A was perpendicularly cleaved and acted as an output coupler, whilst fiber end B (Fig. 2) was angle cleaved at an angle of approximately 10 degrees in order to minimize Fresnel back reflection. The light emitted by the fiber end B was collimated using an MIR Sapphire ball lens with focal length of $\mathrm{f}=6 \mathrm{~mm}$. The cavity was closed by a flat gold-coated high reflector mirror with reflectance $>96 \%$. The output power was measured using a thermal power sensor (S415C Thorlabs). In order to reject the pump wavelength, an optical filter with cut-on of $2.4 \mu \mathrm{m}$ (Edmund Optics \#68659) was placed before the power meter. The output spectrum generated by the fiber laser was monitored using a $150 \mathrm{~mm}$ optical monochromator (MSH-150 LOTQuantum Design $\mathrm{GmbH}$ ) with a diffraction grating blazed at $4 \mu \mathrm{m}$ and coupled to a thermo-electrically cooled MCT detector (Vigo System PVI-4TE-5).

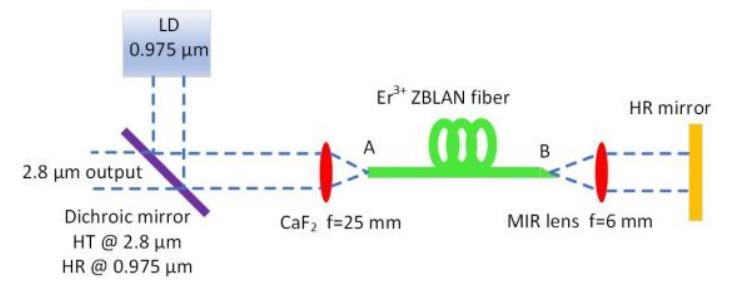

Fig. 2. Schematic diagram of the $\mathrm{Er}^{3+}$ :ZBLAN fiber laser.

Figure 3 shows the dependence of the measured laser output power on the incident pump power for the laser cavity presented in Fig.2. A $24 \%$ slope efficiency, and $2.08 \mathrm{~W}$ maximum output power, was obtained for a 3 meter long $\mathrm{Er}^{3+}$ :ZBLAN fiber. A low laser threshold of around $100 \mathrm{~mW}$ was measured for the developed fiber laser. The obtained slope efficiency is in good agreement with the results previously published in the literature [5]. Higher output powers could be experimentally achieved however, at those output powers the laser behaved unstably, most likely due to rapid $\mathrm{OH}$ in-diffusion into the glass fiber end under heating [9]. Due to possible fiber damage from $\mathrm{OH}$ diffusion the output power was thus limited to $2 \mathrm{~W}$ whilst in the future a complete laser cavity enclosure and purging with dry air or nitrogen is planned.

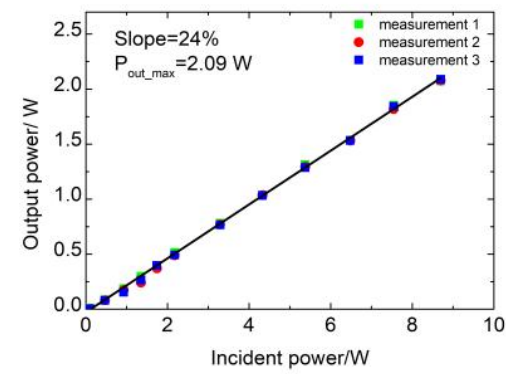

Fig. 3. Output power of $2.8 \mu \mathrm{m} \mathrm{Er}^{3+}$ :ZBLAN fiber laser as a function of incident pump power. 
Figure 4 shows the spectral dependence of the MIR fiber laser emission recorded for different pump power levels. It can be noted that the laser center wavelength shifts towards longer wavelengths with an increase of the pump power. This behavior is typical for a free-running laser where populations of sub-levels in the upper laser level depend on pump power [13]. Figure 4 shows that the laser operates between 2.791 and $2.799 \mu \mathrm{m}$ with FWHM (full width half maximum) bandwidth of around $3 \mathrm{~nm}$. Additionally, the presence of side lobes in the laser spectrum is indicative of ASE (amplified spontaneous emission) signal or second laser peak. In order to fix the laser wavelength, such elements as FBGs (Fiber Bragg Gratings) or an AOTF (acousto-optic tunable filter) should be placed inside the laser cavity [5,13], the commercial availability of which is still very limited at these wavelengths.

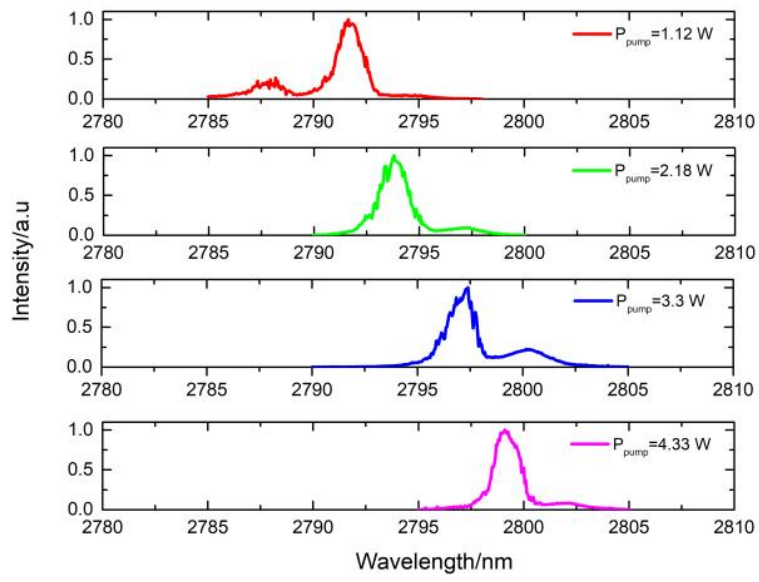

Fig. 4. Measured CW $\mathrm{Er}^{3+}$ :ZBLAN fiber laser output spectrum for different incident pump powers.

Under pumping at $0.975 \mu \mathrm{m}$ strong green luminescence was emitted from the fiber. This green luminescence was collected from the side of the $\mathrm{Er}^{3+}:$ ZBLAN fiber (around 5 $\mathrm{cm}$ from pump launching fiber end A), using a large core (200 $\mu \mathrm{m}$ diameter, NA=0.22) multimode silica fiber, and subsequently analyzed by means of a spectrometer operating between 350-700 nm (CCS 100/M Thorlabs). The recorded spectrum of visible emission pumped at $0.975 \mu \mathrm{m}$ is shown in Fig. 5. Four emission bands centered at $405 \mathrm{~nm}\left({ }^{2} \mathrm{H}_{9 / 2} \rightarrow{ }^{4} \mathrm{I}_{5 / 2}\right), 520 \mathrm{~nm}\left({ }^{2} \mathrm{H}_{11 / 2} \rightarrow{ }^{4} \mathrm{I}_{15 / 2}\right)$, $544 \mathrm{~nm}\left({ }^{4} \mathrm{~S}_{3 / 2} \rightarrow{ }^{4} \mathrm{I}_{15 / 2}\right)$ and $658 \mathrm{~nm}\left({ }^{4} \mathrm{~F}_{9 / 2} \rightarrow{ }^{4} \mathrm{I}_{15 / 2}\right)$ were recorded. Up-conversion mechanisms responsible for visible emission most likely can be attributed to ETU2 (energy transfer up-conversion) or ESA (excited state absorption) (see Fig.1) [9]. Finally, it is noted that the measured visible emission spectrum is in good agreement with the results obtained in $\mathrm{Er}^{3+}$ : ZBLAN glass by other authors [20].

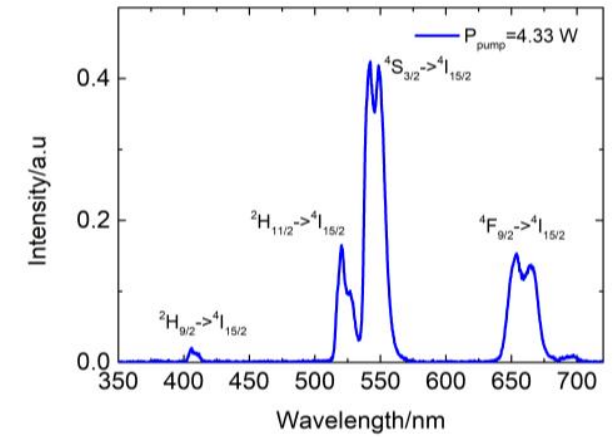

Fig. 5 . Up-converted $\mathrm{Er}^{3+}$ : ZBLAN emission spectrum recorded under $0.975 \mu \mathrm{m}$ pumping.

In conclusion, $\mathrm{Er}^{3+}$ :ZBLAN fiber laser operating at around $2.8 \mu \mathrm{m}$ is reported. A maximum slope efficiency of $24 \%$ and an output power of $2.08 \mathrm{~W}$ with a low laser threshold of $100 \mathrm{~mW}$ were obtained. In the future modifications to the laser cavity arrangements aimed at power scaling will be implemented.

\section{References}

[1] S. D. Jackson, Nature Photonics 6, 423 (2012).

[2] V. Portosi, D. Laneve, C.M. Falconi, F. Prudenzano, Sensors 19, (2019).

[3] M. C. Falconi, D. Laneve, and F. Prudenzano, Fibers 5, 23 (2017)

[4] M. Michalska, P. Grześ, J. Świderski, Phot. Lett. Poland, 11, 109 (2019).

[5] Y.O. Aydin, V. Fortin, R. Vallée, M. Bernier, Opt. Lett. 43, 4542 (2018).

[6] S. Crawford, D.D. Hudson, S.D. Jackson, IEEE Phot. J. 7, 1 (2015).

[7] V. Fortin, F. Jobin, M. Larose, M. Bernier, R. Vallée, Opt. Lett. 44, 491 (2019).

[8] L. Sójka et al., IEEE Photon. Technol. Lett. 30, 1083 (2018).

[9] M. Pollnan, S.D. Jackson, IEEE J. Sel. Top. in Quant. Electron. 7, 30 (2001).

[10] Y.O. Aydin, F. Maes, V. Fortin, S.T. Bah, R. Vallée, M. Bernier, Opt. Expr. 27, 20659 (2019).

[11] C.A. Schäfer et al., Opt. Lett. 43, 2340 (2018).

[12] O. Henderson-Sapir, J. Munch, D.J. Ottaway, Opt. Expr. 24, 6869 (2016).

[13] F. Maes, V. Fortin, S. Poulain, M. Poulain, J.-Y. Carrée, M. Bernier, R. Vallée, Optica 5, 761 (2018).

[14] R.I. Woodward, M.R. Majewski, D.D. Hudson, S.D. Jackson, APL Photonics 4, 020801 (2019).

[15] M. Kochanowicz et al., Opt. Mater. Express 9, 4772 (2019).

[16] M. Kochanowicz et al., Optical Materials 101, 109707 (2020).

[17] J. Wang, X. Zhu, M. Mollaee, J. Zong, N. Peyhambarian, Opt. Expr. 28, 5189 (2020).

[18] M.C. Falconi, D. Laneve, V. Portosi, S. Taccheo, F. Prudenzano, J. Lightwave Technol. 1 (2020).

[19] K. Anders, A. Jusza, P. Komorowski, P. Andrejuk, R. Piramidowicz, J. Lumin. 201, 427 (2018)

[20] P. Komorowski, K. Anders, U. Zdulska, R. Piramidowicz, Photon. Lett. Pol. 9, 85 (2017).

[21] J. Swiderski, M. Michalska, P. Grzes, Appl. Phys. B 124, 152 (2018).

[22] V. Fortin, M. Bernier, S.T. Bah, R. Vallée, Opt. Lett. 40, 2882 (2015). 